\title{
Metodología de diseño de sistemas digitales con reconfiguración parcial dinámica sobre FPGA de bajo costo
}

\section{A design methodology for digital systems using partial run time reconfiguration for low-cost EPGA}

\section{Resumen}

En este documento se presenta una metodología para el diseño de sistemas digitales usando reconfiguración parcial dinámica basada en diferencias en FPGA de la familia Spartan de Xilinx. Se aborda el problema de diseño en alto nivel de abstracción y se muestran las herramientas disponibles para traducir el diseño en un circuito funcional sobre una plataforma autorreconfigurable.

\section{Palabras clave}

Block RAM, FPGA, hardware reconfigurable, reconfiguración parcial dinámica, SelectMAP, sistema digital.

\section{Abstract}

This paper presents a design methodology for digital systems using difference-based runtime partial reconfiguration on low-cost Xilinx Spartan FPGA family. High-level abstraction design issues are described and available tools for translating design in a functional circuit using a self-reconfigurable hardware platform are shown.

\footnotetext{
* Ingeniero Electrónico. Estudiante de Maestría en Ingeniería. - Automatización Industrial, Departamento de Ingeniería Eléctrica y Electrónica, Universidad Nacional de Colombia Bogotá, Colombia.Email: vhbastidasa@unal.edu.co

** Ingeniero Electricista, Magíster en Ingeniería Eléctrica Departamento de Ingeniería Eléctrica y Electrónica, Universidad Nacional de Colombia, Bogotá, Colombia.Email: jseslavag@unal.edu.co
} 


\section{Keywords}

Block RAM, digital system, FPGA, reconfigurable hardware, run-time partial reconfiguration, SelectMAP.

\section{Introducción}

El hardware reconfigurable, usado como plataforma para el diseño de sistemas digitales, permite realizar modificaciones del comportamiento lógico de un circuito usando Lenguaje de Descripción de Hardware (en adelante, HDL por su acrónimo en inglés hardware Description Language). Esta característica, denominada reconfiguración, incrementa la flexibilidad de un sistema digital y otorga al diseñador una herramienta fuerte para modificar, mejorar y optimizar su diseño original [1].

El concepto de flexibilidad de diseño adquiere un nivel superior cuando se utiliza Reconfiguracion Parcial Dinámica (en adelante, RPD). Este tipo de reconfiguración aprovecha la capacidad de ciertas familias de FPGA (Field Programmable Gate Array) de modificar la función de regiones seleccionadas, en cualquier momento después de su configuración inicial [2].

Se han hecho algunos estudios [3], [4], [5], [6], [7] que han podido traducirse exitosamente en diseños de hardware que son capaces de reutilizar recursos que solo son usados ocasionalmente en la operación de un sistema digital. La reutilización de estos recursos de hardware permite hacer mejoras importantes en términos de reducción de espacio, aumento de la velocidad de operación y disminución del consumo de potencia eléctrica [1].

No obstante, la posibilidad de mejoras en la operación de un sistema digital, la RPD no tiene un uso demasiado extendido y varias aplicaciones que podrían aprovechar sus características no hacen uso de ella, principalmente, porque exige un conocimiento profundo de la arquitectura y la tecnología del hardware reconfigurable que se esté usando. Es también una limitante importante el hecho de que el flujo de diseño de la RPD solo esté soportado y bien documentado en los FPGA de las familias Virtex, Artix y Kintex [1], [8] (en lo referente al fabricante Xilinx, únicamente), cuyos precios son más altos.

La RPD puede usarse en un sistema digital de dos formas: basada en diferencias y basada en módulos. La RPD basada en diferencias se enfoca en las estructuras primitivas de hardware contenidas en el FPGA y permite la modificación de sus atributos. La RPD basada en módulos permite modificar, insertar o retirar regiones más grandes de hardware con su descripción funcional.

En este documento se presenta el desarrollo de una metodología de diseño enfocada en aplicar RPD basada en diferencias, en la familia de FPGA de bajo costo Spartan de Xilinx. No se ha incluido la RPD basada en módulos debido a que su implementación requiere de una arquitectura diferente que solo esta disponible en los FPGA Spartan-6 o superiores. La presentación de esta metodología se complementa con la descripción de las herramientas disponibles y necesarias para traducir un diseño en un circuito digital funcional y, como prueba de concepto, se muestra el desarrollo completo de un ejemplo de circuito que aprovecha las propiedades de la RPD basada en diferencias. 
Al no existir un flujo de diseño estándar para trabajar con RPD en FPGA de la familia Spartan, el desarrollo de la metodología de diseño que se presenta introduce la posibilidad de construir sistemas digitales para hardware reconfigurable con las prestaciones de la RPD y la accesibilidad de los FPGA de bajo costo.

La información contenida en este documento se distribuye así: en la sección dos se presenta el análisis y la metodología propuesta para establecer un flujo de diseño completo de un sistema digital usando RPD en FPGA de bajo costo. En la sección tres se detalla el diseño de un circuito en el que se usa la metodología mencionada anteriormente. En la sección cuatro se presentan las conclusiones y el trabajo futuro.

\section{Metodología de diseño}

En la Figura 1 se aprecia un diagrama del procedimiento de diseño constituido por siete etapas en alto nivel de abstracción que son la base de la metodología para construir un sistema digital con RPD basada en diferencias.

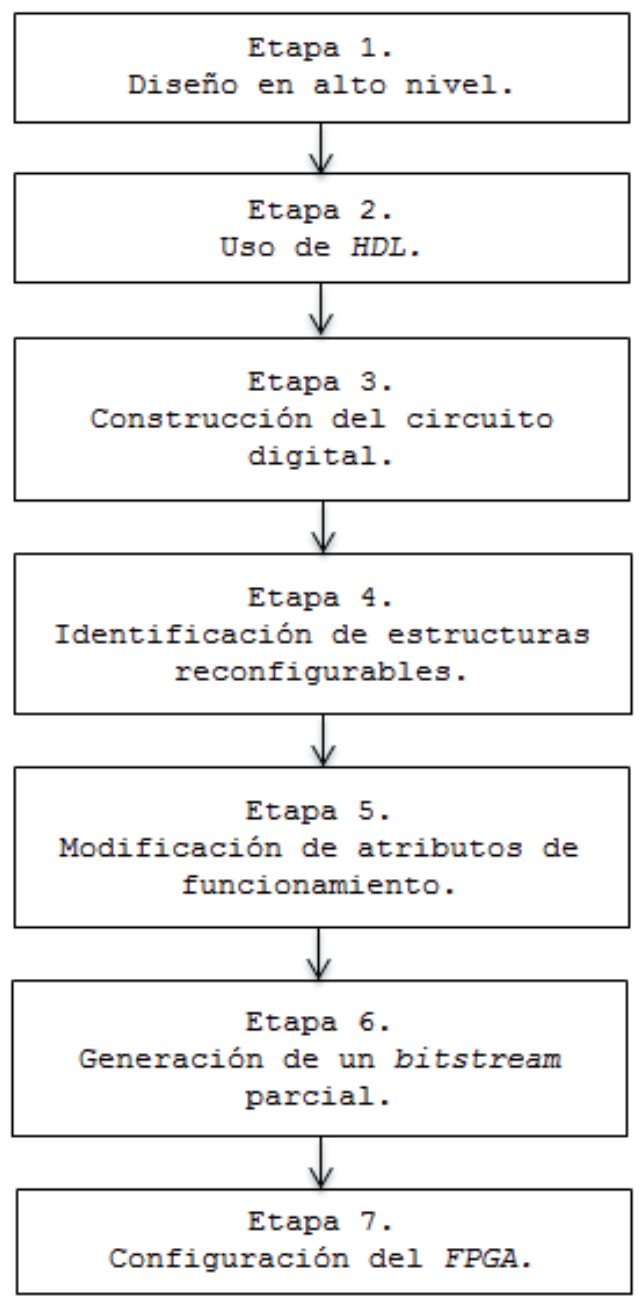

Figura 1. Siete etapas de diseño en alto nivel para construir un sistema digital con RPD basado en diferencias, sobre un FPGA de bajo costo. 


\section{II-A. Uso de las herramientas de diseño}

A continuación se explica cómo se usan las herramientas de software en cada una de las etapas de diseño:

II-A1. Diseño en alto nivel: el proceso de diseño inicia con una idea de un sistema digital en el que se incluyen regiones reconfigurables dinámicamente, cuyas funciones pueden predefinirse con claridad. Se espera llegar al punto de obtener estructuras primitivas físicas (se debe considerar que, dependiendo de la arquitectura de FPGA, las modificaciones mínimas pueden estar restringidas a regiones más grandes), principalmente slices, que se encuentran dentro de cada celda lógica de un FPGA.

Los circuitos digitales que pueden aprovechar las características de la RPD en FPGA de bajo costo se centran generalmente en modificaciones de las funciones lógicas atribuidas a cada slice o al comportamiento predeterminado de una estructura primitiva, por ejemplo, un Digital Clock Manager (en adelante, DCM) o un multiplicador. Los sistemas que funcionan con base en señales de reloj variables pueden aprovechar también esta característica.

II-A2. Uso de HDL: cuando se tiene una idea clara del sistema que se va a diseñar, es conveniente realizar una primera división, muy específica del sistema: en aquellas regiones estáticas, que no van a modificarse después de la primera configuración, puede usarse cualquier tipo de descripción de hardware; en las regiones diseñadas para reconfigurarse dinámicamente, es altamente recomendado el uso de la descripción estructural (en bajo nivel) de cada módulo.

Las consideraciones en el tipo de descripción mencionadas recientemente son de gran ayuda porque en el proceso de Síntesis se pueden traducir directamente a hardware, estructuras bien definidas, con ubicación y conexionado fijo. Es entonces mucho más fácil acceder posteriormente a realizar modificaciones en los atributos lógicos de dichas estructuras.

II-A3. Construcción del circuito digital: Al finalizar la etapa de diseño HDL, el proceso de Síntesis permitirá observar el esquemático RTL que debe contener las estructuras reconfigurables. Si todo está bien, el siguiente proceso, la Implementación permitirá que el esquemático se traduzca en hardware. En este punto se debe prestar atención al proceso de Place \& Route. Los resultados de este proceso pueden apreciarse con la herramienta de software FPGA Editor de Xilinx Toools.

II-A4. Identificación de estructuras reconfigurables: FPGA Editor ofrece una vista con la lista de componentes que han sido exitosamente ubicados y conectados dentro del FPGA; se debe buscar en esta lista aquel componente que se desee modificar. Facilita el trabajo de identificación, el hecho de que cada estructura conserve el mismo nombre con el que aparece en el esquemático RTL en el proceso de Síntesis.

II-A5. Modficación de atributos de funcionamiento: cuando se ha seleccionado la estructura que va a modificarse se puede entrar al modo de edición y revisar la información de sus atributos lógicos. Dependiendo de la estructura, se pueden modificar atributos como la función lógica, el periodo y la frecuencia de operación, el factor de multiplicación de frecuencia, la fase, las entradas activas, entre otros. 
II-A6. Generación de un bitstream parcial: la estructura modificada debe guardarse como un diseño nuevo con un nombre diferente, con extensión .ncd (Native Circuit Design). Esto permitirá después, con la herramienta Bitgen, de Xilinx Tools, realizar una comparación entre el archivo de configuración bitstream original (.bit) y el diseño modificado (.ncd), para generar un nuevo archivo de configuración bitstream. Este archivo contiene solo información de las modificaciones, por lo cual es pequeño y notablemente más fácil de manejar que un bitstream completo.

II-A7. Configuración del FPGA: cuando se regenera el archivo bitstream completo, el archivo bitstream parcial queda contenido en él, lo que significa que se puede realizar la configuración del FPGA como se hace convencionalmente, por ejemplo vía JTAG. La información que se introduce en el dispositivo reconfigurable es suficiente para que en adelante se auto-reconfigure, sin necesidad de ninguna conexión externa.

El procedimiento completo mencionado anteriormente puede repetirse varias veces. La RPD no está limitada a una sola modificación, por lo cual cada bitstream parcial nuevo puede contener modificaciones aplicables a la misma estructura, o incluso, a estructuras nuevas.

\section{II-B. Requerimientos de hardware para RPD}

La RPD puede implementarse en dispositivos FPGA de bajo costo a través del modo SelectMAP (también conocido como Slave Parallel). SelectMAP es un modo de configuración que permite conectar hasta tres dispositivos Xilinx y posibilita su lectura y escritura en paralelo [10]. La Figura 2 muestra las conexiones entre la interfaz SelectMAP y los periféricos necesarios para controlar el proceso de RPD.

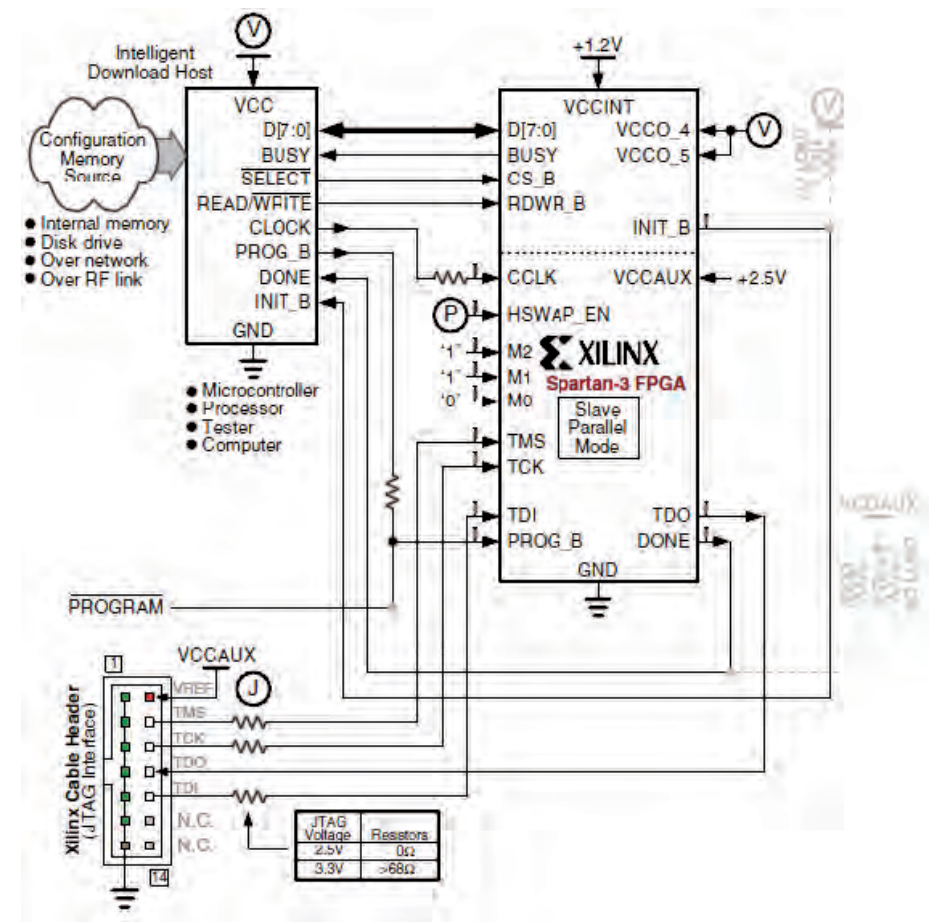

Figura 2. Esquemático del conexionado de SelectMAP para un FPGA XC3S200. Este esquemático está diseñado originalmente para ser controlado mediante periféricos.

Fuente: tomado de [9]. 
Los bitstreams parciales previamente generados deben convertirse a un formato adecuado, y posteriormente, deben almacenarse en una memoria. El Gestor de RPD, que puede ser un procesador, un microcontrolador, una PC o, incluso, un módulo diseñado internamente en un FPGA, es responsable de controlar la sincronización, la secuencia de inicio y finalizar la comunicación con la interfaz SelectMAP. Otras funciones del Gestor de RPD son generar señales de reloj adecuadas y transmitir cada byte desde la memoria hasta SelectMAP.

\section{Utilización de la metodología}

Con el fin de aplicar la metodología propuesta y como ejemplo de un módulo reconfigurable, se presenta el proceso completo del diseño de un gestor dinámico de señales de reloj (Dynamic clock manager), tomando como base un FPGA Spartan-3. La gestión dinámica de las señales de reloj es usada frecuentemente como un método para reducir el consumo de potencia en diseños de hardware, al cambiar la frecuencia de operación dependiendo de las necesidades.

El proceso de diseño empieza por identificar las estructuras adecuadas y disponibles en el FPGA. El FPGA Spartan-3 de Xilinx dispone de cuatro módulos DCM. Estos módulos son estructuras fijas, con funciones claramente determinadas, entre las que se encuentra la variación de frecuencia. Es esta la parte reconfigurable del sistema; cualquier circuito puede aprovechar la variación de frecuencia de este módulo, por lo tanto, el diseño se limitará solo al módulo DCM.

Un módulo DCM puede instanciarse usando descripción estructural y el proceso de Síntesis resulta en el esquemático RTL que se muestra en la Figura 3. En este caso, se ha usado un FPGA XC3S200.

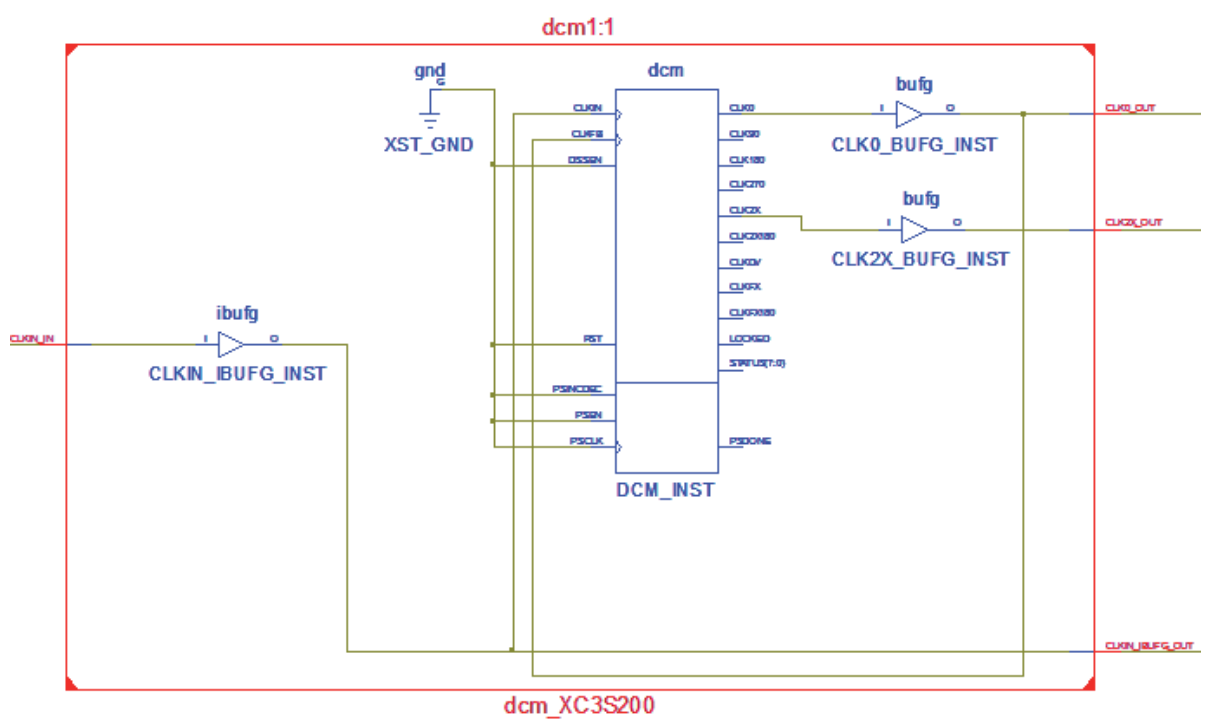

Figura 3. Esquemático RTL obtenido después del proceso de Síntesis de un módulo DCM. La Síntesis de esta estructura primitiva garantiza que el componente estará disponible en la etapa de Implementación y podrá ser modificado. 
Después de realizar el proceso de Implementación, se usa FPGA Editor para apreciar los resultados de Place \& Route y encontrar el componente dcm_XC3S200. Cuando se activa el modo de edición, los atributos del módulo DCM pueden visualizarse, como se muestra en la Figura 4.

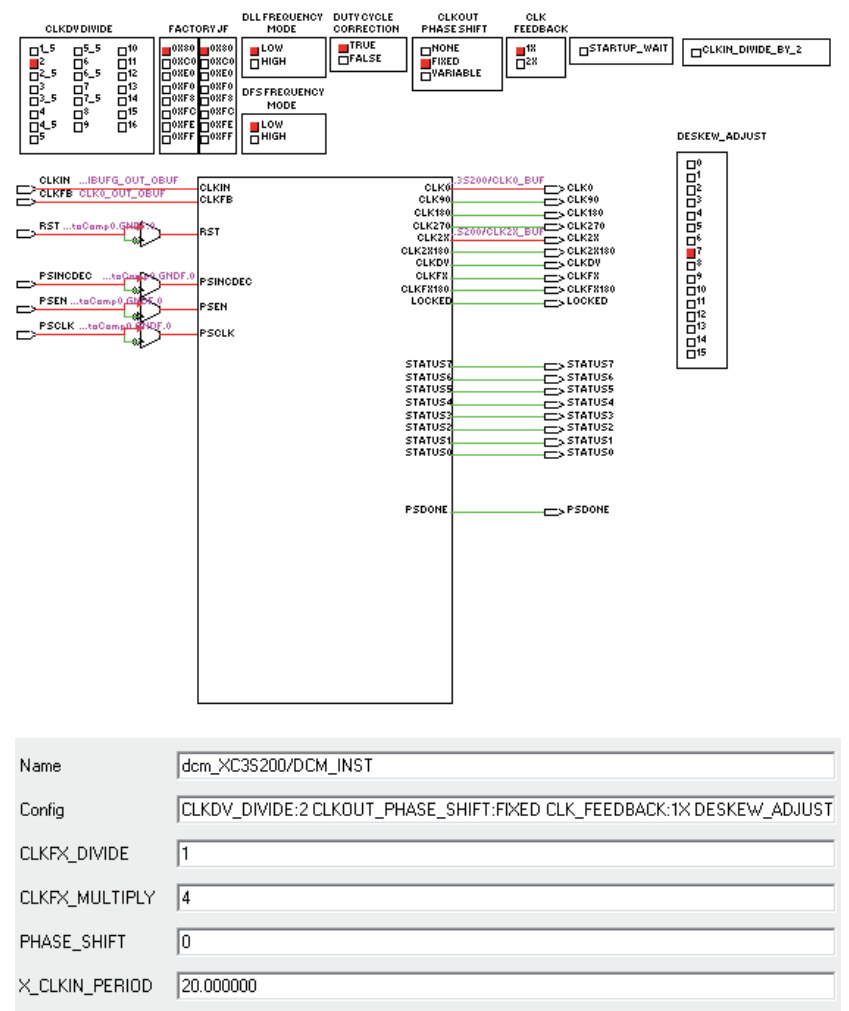

Figura 4. Atributos modificables en uno de los cuatro módulos DCM disponibles dentro del FPGA XC3S200.

Si el atributo X_CLKIN_PERIOD, originalmente fijado en $20 \mathrm{~ns}$, se cambia, la frecuencia (o el periodo) puede modificarse a cualquier valor menor que la frecuencia inicial de 50 $\mathrm{MHz}$. Cada atributo puede modificarse las veces que sea necesario y pueden generarse, también, varios bitstreams parciales.

\section{III-A. Sustitución de periféricos por módulos de hardware}

En la Figura 2 se muestran las conexiones de la interfaz SelectMAP con los periféricos necesarios para controlar el proceso de RPD. El concepto del sistema auto-reconfigurable es que ningún periférico sea necesario y que el FPGA tenga la posibilidad de controlar su propia reconfiguración. Dadas sus características, el FPGA puede ser aprovechado para sustituir a estos periféricos de la siguiente forma:

III-A1. Memoria: dentro de los FPGA de la familia Spartan se encuentran conectados físicamente varios bloques de memoria que no son utilizados con mucha frecuencia debido a su tamaño reducido. Esta memoria, conocida como Block RAM, a diferencia de una celda lógica solo tiene la funcion de almacenamiento, no tiene funciones adicionales. Por lo anterior, considerando el tamaño de un archivo de configuración bitstream parcial de un FPGA, la Block RAM puede destinarse exclusivamente para este fin. 
III-A2. Procesador: Este periférico se usa para controlar la secuencia de configuración del FPGA a través de SelectMAP. Existen algunas señales de control, para este caso son únicamente 2, una señal de reloj y 8 líneas de datos que permiten cargar de forma paralela, un byte a la vez, la información correspondiente a un archivo de configuración bitstream. Aunque la mayoría de los diseños contemplan el uso de un procesador físico, las tarjetas de desarrollo basadas en FPGA más recientes (Spartan-6 o superiores) cuentan con un módulo denominado Internal Configuration Access Port (ICAP) que es un circuito cuyo comportamiento es un espejo del funcionamiento de SelectMAP y permite interactuar completamente con este modo de configuración. Como el módulo ICAP no existe en el FPGA que se usó para las pruebas, y con base en el concepto original de [7], se diseñó un circuito sencillo al que se llamó Gestor de RPD, que permite cargar la información de la Block RAM, enviarla a SelectMAP y que a su vez, no usa demasiados recursos de hardware. Con la ayuda adicional del procedimiento usado en [9], se determina que la función principal, la de control, se satisface si se cumplen los siguientes pasos:

1. Garantizar que CS_B vaya a un nivel lógico bajo.

2. Garantizar que RDWR_B vaya a un nivel bajo, al menos un ciclo de reloj después.

3. A partir del siguiente ciclo de reloj, SelectMAP empieza a recibir cada byte de información.

4. Sincronizar la memoria con SelectMAP hasta que se envíe la información completa del bitstream.

5. Esperar por lo menos 8 ciclos de reloj para finalizar la comunicación.

6. Enviar CS_B a un nivel lógico alto.

7. Al menos un ciclo de reloj después, enviar RDWR_B a un nivel lógico alto.

CS_B corresponde a la señal de Chip_Select y RDWR_B a la señal que permite iniciar el proceso de escritura en SelectMAP. Existen muchas más señales que, para este caso, se pueden omitir o fijar, y otras que son manejadas internamente por el FPGA cuando reconoce que se está trabajando en modo SelectMAP.

En la Figura 5 se observa el funcionamiento del Gestor de RPD que reproduce el comportamiento mencionado anteriormente. Se muestra un ciclo de configuración completo con un archivo bitstream parcial que se usó como ejemplo; la secuencia 00, 00, 00, b0, corresponde a la finalización del archivo e indica que se trata de un bitstream parcial.

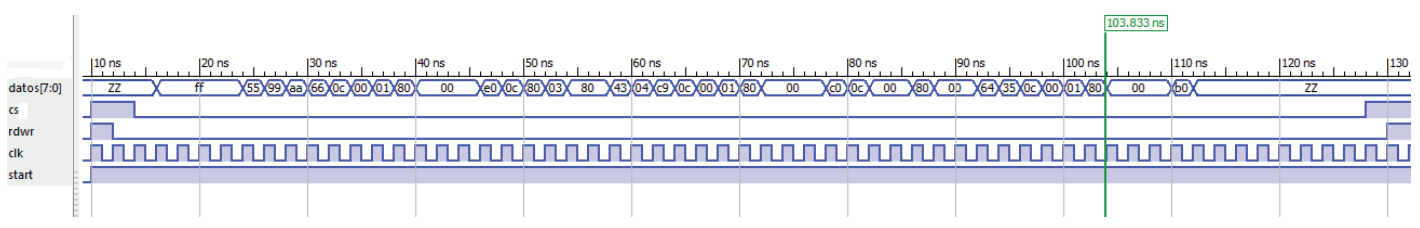

Figura 5. Ciclo de reconfiguración completo usando el Gestor de RPD para controlar el modo SelectMAP. Solo se necesitan dos líneas de control, una línea de reloj y ocho líneas de datos. La señal adicional start fue incluida para garantizar que la reconfiguración se hiciera una sola vez.

El uso de los módulos mencionados permite que el sistema sea completamente autoreconfigurable. Solo se necesita que desde una PC se realice la configuración inicial que incluya 
al circuito Gestor de RPD, una Block RAM debidamente diseñada y dimensionada, y que dentro de esta se ubique adecuadamente la información de los archivos bitstream parciales.

\section{III-B. Tratamiento de los archivos de configuración}

Un archivo de configuración completo contiene la información total de las conexiones, ubicación y de la lógica que se necesitan para que cierto diseño pueda construirse dentro de un FPGA. Este archivo bitstream tiene un tamaño fijo para cada FPGA, independientemente de que el diseño sea una sola compuerta lógica o algo muy complejo [9]. En el caso del FPGA XC3S200 el archivo de configuración completo tiene un tamaño de 1047616 bits, demasiado grande para almacenarse en la Block RAM. Sin embargo, al trabajar con archivos bitstream parciales, cuya información se limita a las diferencias con respecto a un bitstream original, su tamaño es mucho menor.

El primer paso consiste en obtener un bitstream parcial. La herramienta Bitgen genera un archivo bitstream parcial comparando el archivo .bit original y el archivo .ncd modificado después de la etapa de Implementación. Bitgen está disponible desde TCL Console, por lo tanto se hace necesario introducir el siguiente comando:

\section{bitgen -w -g ActiveReconfig:Yes -g Persist:Yes -g -r original.bit modificado.ncd}

El resultado es el archivo bitstream parcial modificado.bit, mucho más pequeño que el archivo original, pero al estar basado en este último, no afecta a la configuración inicial en regiones no modificadas.

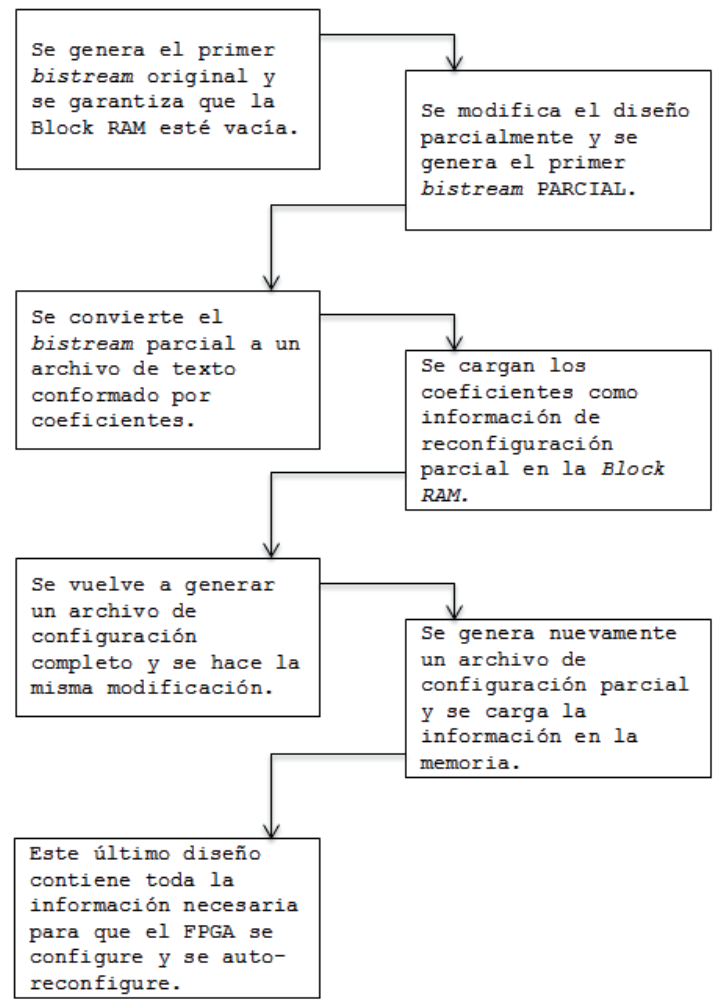

Figura 6. Procedimiento de seis etapas que debe usarse para generar un archivo bitstream parcial correcto. 
Se requiere repetir el proceso de Implementación para evitar el problema de recurrencia que aparece por usar un bitstream completo que contiene un bitstream parcial.

Usando iMPACT de Xilinx Tools se puede convertir el archivo bitstream en un archivo hexadecimal con byte-swapping. Este procedimiento se hace necesario debido a que SelectMAP está diseñado para ser controlado con un procesador externo y se debe mantener coherencia entre los bits más y menos significativos de cada byte. Después, usando el software Coegen 3.0, desarrollado específicamente para este estudio por no existir ninguna herramienta similar disponible, se convierte el archivo .hex en un archivo .coe que puede cargarse directamente como información en Block RAM.

Un problema adicional que se debe solucionar antes de seguir con el diseño, es que se está tratando con un bitstream completo que está conteniendo un bitstream parcial; esto genera inicialmente un problema de recurrencia que se puede solucionar siguiendo el procedimiento de la Figura 6 que se modificó para adaptarlo a las necesidades del proyecto, con base en la información presentada en [7].

\section{Conclusiones y trabajo futuro}

La metodología de diseño que se muestra en este documento permite estandarizar el proceso de construcción de un circuito digital con RPD basada en diferencias en FPGA de bajo costo. Aunque el diseño digital en alto nivel de abstracción sigue dependiendo de las habilidades del diseñador y exige que este tenga conocimientos profundos, tanto de sistemas digitales como de arquitectura de FPGA, la metodología aquí presentada ayuda a solucionar un problema crítico del diseño: definir las regiones reconfigurables y realizar modificaciones dinámicas con seguridad en hardware que no fue disenado originalmente para usar RPD.

La plataforma autorreconfigurable requiere el uso de la memoria por bloques interna del FPGA. En este aspecto la mayor limitación es, por mucho, la cantidad de los bitstreams que pueden almacenarse internamente. Dependiendo del FPGA que se seleccione, es probable que su memoria interna no tenga capacidad mayor que la de almacenar un único archivo de configuración. Este no es un problema que pueda resolverse seleccionando un FPGA con una Block RAM más grande, puesto que un FPGA de mayor tamaño requiere de un bitstream de mayor tamaño también.

La etapa que presenta mayor dificultad al aplicar la metodología de diseño es la generación de bitstreams parciales. Fue necesario desarrollar software que completara el procedimiento y elaborar un flujo de diseño que se adaptara al tratamiento que se le estaba dando a los archivos de configuración. La solución a los inconvenientes de esta etapa, que se describe en la sección III-B garantiza la integridad de los archivos bitstream parciales.

El trabajo futuro se enfoca en mejorar el proceso de modificación de las estructuras primitivas específicas de cada arquitectura. Adicionalmente, se desarrollarán sistemas digitales más complejos para establecer en un espectro más amplio, las ventajas, 
desventajas y limitaciones de la RPD basada en diferencias en dispositivos de bajo costo. Se estudiarán tambien las consideraciones de diseño de la RPD basada en módulos que permite diseñar regiones reconfigurables más grandes y complejas, usando principalmente su descripción funcional.

\section{Referencias}

[1] Xilinx Corporation, Partial reconfiguration user guide, UG702, v12.1. Webpage: http:www.xilinx.comsupportdocumentationsw_manualsxilinx12_1ug702. pdf. 2010.

[2] L. Wang and F. Wu, Dynamic partial reconfiguration in FPGAs, vol. 2, pp. 445-448. Third international symposium on intelligent information technology application, 2009.

[3] C. Inabla and K. Arshak, Using dynamic partial reconfiguration approach to read sensors with different bus protocol. SAS IEEE Sensors Applications Symposium. New Orleans, LA, USA, 2009.

[4] F. Ghaffari, B. Miramond and F. Verdier, Dynamic adaptation of hardware-software scheduling for reconfigurable Systemon-Chip. The 19th IEEE/IFIP International Symposium on Rapid System Prototyping. Monterrey, California, USA, 2009.

[5] J. Cardoso, J. Basilio Simoes, C. Correia, A. Combo, R. Pereira, J. Sousa, N. Cruz, P. Carvalho and C. Varandas, A high performance reconfigurable hardware platform for digital pulse processing, vol. 51, No. 3. IEEE Transactions on nuclear science, 2004.

[6] H. Kopka and A. Yurdakul, A self-reconfigurable platform for general purpose image processing systems on low-cost Spartan-6 FPGAs. Disponible en: http://www.cmpe.boun.edu.tr.

[7] H. Kopka and A. Yurdakul, Dynamic partial self-reconfiguration on Spartan-iii FPGAs via a parallel configuration access port (PCAP). Disponible en: http:// www.cmpe.boun.edu.tr.

[8] D. Dye, Partial reconfiguration of Xilinx FPGAs using ISE Design Suite, WP3742, v. 1.2. Disponible en: http://www.xilinx. comsupportdocumentationwhite_paperswp374_partial_Reconfig_Xilinx_FPGAs.pdf, 2012.

[9] Xilinx Corporation, Spartan-3 generation configuration user guide. Extended Spartan-3A, Spartan-3E and Spartan-3 FPGA families, UG332, v. 1.6. Disponible en: http://www.xilinx.comsupportdocumentationuser_guidesug332.pdf, 2009.

[10] Xilinx Corporation, Disponible en: http://www.xilinx.comitpxilinx10isehelppim_c_introduction_SelectMAP.htm.

UNIVERSIDAD DE SAN BUENAVENTURA BOGOTÁ
CUPÓN DE SUSCRIPCIÓN

\section{INGENIUM}

\section{Revista de la Facultad de Ingeniería}

Fecha: Día Suscriptor

Apelidos

Nombres

\begin{tabular}{|l|l|l|}
\multicolumn{2}{c}{ Residencia } \\
\hline Dirección & \multicolumn{3}{c|}{} \\
\hline Ciudad & Fax & \multicolumn{2}{c}{ País } \\
\hline Teléfono & & Celular \\
\hline
\end{tabular}

E-mail

\section{Suscripción anual}

\begin{tabular}{ll|l} 
Colombia $\$ 120.000,00 \quad$ Exterior
\end{tabular}

Mes

\begin{tabular}{|c|c|c|c|}
\hline \multicolumn{2}{|c|}{ Número suelto } \\
\hline Colombia & $\$ 60.000,00$ & Exterior & US $\$ 35,00$ \\
\hline & \multicolumn{2}{|c|}{ Suma enviada: } \\
\hline
\end{tabular}

\section{Suscripción a partir del número:}

- Consigne en la cuenta de ahorros número 605-07721-3 del Banco de Bogotá, a nombre de la Universidad de San Buenaventura, Bogotá, D. C.

- Envíe copia del recibo de consignación, junto con este cupón, a la Editorial Bonaventuriana (Fax: 677 3003).

- Si lo prefiere presente el cupón y el recibo de consignación directamente en la Editorial Bonaventuriana

(Cra. 8 H 172-20, Bogotá, D. C., Colombia). 